\title{
Excess epicardial fat volume in post- menopausal women is associated with increased cardiovascular structural and functional abnormalities
}

\author{
Mahfouz El Shahawy $^{1 *}$, Sukanya Mohan ${ }^{1}$ and Antonella Sabatini ${ }^{1,2}$ \\ ${ }^{1}$ Cardiovascular Center of Sarasota, Sarasota, Florida 34239, USA \\ ${ }^{2} \mathrm{ASMD}$, Florence, Italy
}

\begin{abstract}
Epicardial fat volume (EFV) has been associated with an increase in cardiovascular structural and functional abnormalities (CVSFA). The purpose of the study is to assess whether a stepwise excess increase in EFV is associated with a proportional increase in CVSFA. We screened 276 asymptomatic individuals, ages 50 to 89 , for cardiovascular disease (CVD) risk using the Rasmussen Risk Score (RRS) and a CT scan for the coronary artery calcium score (CACS) and EFV determination. The RRS scoring system consists of 10 tests; 7 of which are vascular and 3 are cardiac. Additional tests included fasting blood sugar, fasting lipid profile, CRP, ProBNP, waist circumference, and BMI. Of the 276 total subjects that were asymptomatic of cardiac symptoms, 144 were post-menopausal women (52.2\%). These 144 female subjects were further divided into 5 groups based on their EFV: Group 1 (31 subjects) with an EFV <69 $\mathrm{cm}^{3}$, Group 2 ( 45 subjects) with an EFV between 70 $\mathrm{cm}^{3}$ and $94 \mathrm{~cm}^{3}$, Group 3 (21 subjects) with an EFV between $95 \mathrm{~cm}^{3}$ and $119 \mathrm{~cm}^{3}$, Group 4 (33 subjects) with an EFV between $120 \mathrm{~cm}^{3}$ and $144 \mathrm{~cm}^{3}$, and $\mathrm{Group}$ 5 (14 subjects) with an EFV of $145 \mathrm{~cm}^{3}$ and greater. The statistical analysis used to determine statistical significance of the study results included ANOVA, T-Test and Chi-Square analysis. Based on our data, excess epicardial fat volume is associated with earlier structural and functional abnormalities in comparison to normal epicardial fat volume levels.
\end{abstract}

\section{Background}

Epicardial fat is defined as the adipose tissue which directly overlies the heart. It can cover up to $80 \%$ of the myocardium and account for up to $20 \%$ of the heart's mass $[1,2]$. Epicardial fat is directly vascularized by branches of the coronary arteries, and lacks a fascial layer separation from the myocardium, implying a close and strong interaction with both tissue structures [1]. The physiologic function of epicardial fat is complex and not yet completely understood, but can be generally distinguished by various mechanical, metabolic, thermogenic, and endocrine/paracrine functions [1,3]. Additionally, there is growing evidence that human epicardial fat produces bioactive cytokines which are both locally and systemically involved in the regulation of endothelial function. In menopausal women with endothelial dysfunction, menopausal transition is associated with increased carotid arterial stiffness and epicardial fat thickness, independent of age [2].

Epicardial fat volume (EFV), also referred to as epicardial adipose tissue (EAT), has been recently reported to be a novel cardiovascular risk marker [1,4,5]. Early detection of excess EFV (i.e.,> $69 \pm 20$ in females and males) has been found to correlate with early detection of cardiovascular structural and functional abnormalities among subjects with various comorbidities, such as type 2 diabetes and obesity, which mandates treatment through lifestyle modifications and aggressive medical therapy [4]. Epicardial fat belongs to the category of perivascular adipose tissue which also includes the fat surrounding the renal arteries. Epicardial adipose tissue differs from abdominal visceral adipose tissue mainly in its mRNA. Epicardial adipocytes are also smaller than that of abdominal visceral adipocytes $[3,6]$. It has been reported that many obese and/or diabetic individuals have increased EFV, which has been associated with many risk factors contributing to coronary artery disease (CAD) [5,7-9]. Additionally, studies have found an association between excess EFV and metabolic syndrome [10,11]. EFV has also been correlated with other independent cardiac biomarkers, such as high levels of C-reactive protein, BNP, and microalbuminuria, along with cardiovascular risk factors (i.e., hypertension, dyslipidemia, and hyperglycemia) [12]. It has also been associated with cardiovascular structural and functional abnormalities, such as an abnormal rise in blood pressure post-mild protocol exercise, carotid intima-media thickness (CIMT), and left ventricular hypertrophy [13,14]. Under normal physiological conditions, epicardial fat is known to produce antiinflammatory and anti-atherosclerotic cytokines, such as adiponectin and adrenomedullin, for cardioprotective function including increased free fatty acid oxidation, nitric oxide synthesis, and vasodilation $[15,16]$. A decrease in nitric oxide availability and vasodilator imbalance has been linked to the development of microvascular disease-based angina. Accordingly, it is time to focus on epicardial fat pathophysiology as an important risk marker for cardiovascular disease (CVD).

Women usually reach menopause between the ages of 40 and 60 . Although there has not been sufficient data that menopausal women

${ }^{\star}$ Correspondence to: Mahfouz El Shahawy, Cardiovascular Center of Sarasota, Sarasota, Florida 34239, USA, E-mail: mshahawy@cardiologycenter.net

Key words: excess epicardial fat volume, asymptomatic females, microvascular disease, post-menopausal females

Received: January 21, 2021; Accepted: February 03, 2021; Published: February 12,2021 
tend to gain weight, it has been found that postmenopausal women have a greater amount of abdominal visceral fat in comparison to premenopausal women. EFV has been shown to be greater after menopause and may be regulated due to endogenous estrogen $[8,17]$. The increase in EFV after menopause has been correlated with the acceleration of atherosclerosis. Hormone therapy may have positive results in slowing down the deposition of epicardial fat [13]. This study aims to assess whether an Excess EFV in post-menopausal women leads to higher levels of vascular and cardiac abnormalities.

\section{Methods}

We screened 276 asymptomatic individuals, ages between 5089 , for cardiovascular disease (CVD) risk using the Rasmussen Risk Score (RRS) [8], CT scan for coronary calcium scores and epicardial fat volume determination. The RRS consists of 10 tests: 7 of these tests are vascular and 3 are cardiac. The vascular components are large $(\mathrm{C} 1)$ and peripheral (C2) artery stiffness, blood pressure (BP) at rest and post-mild protocol exercise (PME) consisting of a 3-minute walk at $7 \%$ elevation and a speed of $2.5 \mathrm{mph}$. Abnormal BP-PME is defined as a systolic rise of $>30 \mathrm{mmHg}$ compared to systolic BP at rest $[19,20]$. Large (C1) arterial stiffness refers to conducting arteries which store blood ejected from the heart during systole and expel blood to the peripheral tissues during diastole. Peripheral (C2) arteries refer to resistance muscular arteries which can alter vascular smooth muscle tone, allowing them to modulate the velocity of pressure wave [21]. Normotension, elevated BP, and hypertension are defined according to the current ACC/AHA guidelines [22]. Other vascular assessments include Carotid Intima Media Thickness (CIMT), abdominal aorta ultrasound, retinal photography, and microalbuminuria. The 3 cardiac components are Pro-BNP, ECG, and left ventricle ultrasound (LVUS). All EKG abnormalities are considered, but some of these abnormalities include atrial fibrillation, bundle branch block, atrial flutter, etc. The LVUS looks for abnormalities in LV ejection fraction and mass. Additional tests are waist circumference, BMI, fasting blood sugar, fasting lipid profile, and hs-CRP [20]. Each of the 10 tests that make up the RRS is scored as Normal, Borderline, or Abnormal. A normal score $=0$, a borderline score $=1$, and an abnormal score $=2$. After adding up the scores from all 10 tests, a subject can get a score between 0 through 20. A score of between 0-2 indicates low risk of CVD, and a score between 3-5 indicates intermediate risk of CVD, and a score $\geq 6$ indicates strong risk of CVD. The RRS has been a previously peer-reviewed risk score $[19,20]$. Of the 276 total subjects that were asymptomatic of cardiac symptoms, which includes chest pain and other pre-existing cardiac conditions, 144 post-menopausal women $(52.2 \%)$ underwent cardiac CT for coronary artery calcium scoring (CACS) and EFV determination using the Siemens Somatom Definition Dual-source CT scanner 64x2. Quantifying the EFV is done using a non-contrast $3 \mathrm{~mm}$ sequenced scan with cardiac gating and post processing on Siemens VIA workstation. The coronary calcium artery is quantified using the Agatston method. The epicardium fat volume was quantified using Hounsfield method. These 144 female subjects were further divided into 5 groups based on their EFV: Group 1 (31 subjects) with an EFV $<69 \mathrm{~cm}^{3}$, Group 2 (45 subjects) with an EFV between 70 $\mathrm{cm}^{3}$ and $94 \mathrm{~cm}^{3}$, Group 3 (21 subjects) with an EFV between $95 \mathrm{~cm}^{3}$ and $119 \mathrm{~cm}^{3}$, Group 4 (33 subjects) with an EFV between $120 \mathrm{~cm}^{3}$ and 144 $\mathrm{cm}^{3}$, and Group 5 (14 subjects) with an EFV of $145 \mathrm{~cm}^{3}$ and greater. The first group were chosen based on what was considered normal EFV (69 \pm 20 in females) [23] and each subsequent group is an increase of $24 \mathrm{~cm}^{3}$ of EFV. The statistical analysis used to determine statistical significance of the study results included ANOVA, T-Test and Chi-Square analysis. A P-value $<0.05$ was considered statistically significant.

\section{Results}

The baseline characteristics of the groups included individuals who were asymptomatic of cardiac issues such as chest pain, myocardial infarctions, and strokes. All the groups consisted of post-menopausal women who were mostly of the Caucasian race. As noted in Tables 1, 2 and 3 a significant p-value was determined between the average RRS (0.022), abnormal C1 percentage $(<0.0001)$, abnormal C2 percentage $(<0.0001)$, abnormal CIMT percentage $(0.00032)$, average triglyceride levels (0.0011), average microalbumin level (0.0447), average blood sugar level (0.0030), and average HDL level (0.0000393) to their corresponding levels of EFV. The data also reveals that excess epicardial fat (groups 3,4, and 5) is associated with earlier structural and functional abnormalities in comparison to normal epicardial fat volume (groups 1 and 2) such as abnormal endothelial function, CIMT, triglyceride levels, microalbumin levels, blood sugar levels, and HDL levels.

\section{Discussion}

Several methods of measuring cardiac fat volumes are feasible, such as total intrathoracic fat volume and epicardial adipose tissue and thoracic fat (i.e., ITFv, EATv, and TF volume) which can be measured directly by non-contrast cardiac computed tomography [24]. Some studies have utilized echocardiography as a way for quantifying EFV $[2,25]$ but it is more accurately done using computed tomography. Given the high spatial resolution of CT and that adipose tissue has distinct attenuation values, EFV is readily identified allowing for accurate volumetric measurement [6]. Fat attenuation and volume can also be reliably measured from pre-contrast calcium scans, with volume quantification showing particularly strong correlation [26]. It is one of the more reliable and reproducible methods for estimating the extent of epicardial fat volume with high spatial resolution [27]

According to the present study normal EFV (Group 1 and 2) is associated with minimal cardiovascular structural and functional abnormalities. Upon reviewing the data, groups 1 and 2 do not have as many structural and functional cardiovascular abnormalities in comparison to the excess epicardial fat volume groups. Excess EFV (Group 3,4 and 5) is associated with statistically significant cardiovascular structural and functional abnormalities, as well as CVD biomarkers, such as: $\mathrm{C} 2$ abnormalities, abnormal rise in systolic BP, CRP, BNP, and microalbuminuria. As noted in Tables 1, 2 and 3, a significant $\mathrm{p}$-value was determined between the average RRS, abnormal $\mathrm{C} 1$ percentage, abnormal C2 percentage, abnormal CIMT percentage, average triglyceride levels, average microalbumin level, and average HDL level to their corresponding levels of EFV. Studies have found an association between excess EFV and metabolic syndrome [10,11]. EFV has also been correlated with other independent cardiac biomarkers, such as high levels of C-reactive protein, BNP, and microalbuminuria, along with cardiovascular risk factors (i.e., hypertension, dyslipidemia, and hyperglycemia) [12]. The data also reveals that excess epicardial fat (groups 3,4, and 5) is associated with more early cardiovascular diseases risk markers in post-menopausal women.

Normal EFV is associated with minimal cardiovascular structural and functional changes, while excess EFV is associated with statistically significant cardiovascular structural and functional abnormalities, as well as the presence of CVD biomarkers, such as: C2 abnormalities, abnormal rise in systolic BP, CRP, and microalbuminuria. These microvascular changes have been observed and reported on in previous publications over the last decade [19]. EFV appears to be a significant factor involved in microvascular disease due to its ability to secrete several cytokines, referred to as adipokines. These cytokines play an 
Table 1. EFV and structural \& functional abnormalities

\begin{tabular}{|c|c|c|c|c|c|c|}
\hline $\mathbf{E F V}\left(\mathrm{cm}^{3}\right)$ & Average RRS & $\begin{array}{c}\text { Average Resting BP } \\
(\mathrm{mmHg})\end{array}$ & Average CAC & Abnormal C1 & Abnormal C2 & Abnormal CIMT \\
\hline$<69$ & 6 & $137 / 77$ & 133.74 & $52 \%$ & $65 \%$ & $42 \%$ \\
\hline $70-94$ & 5 & $137 / 75$ & 210.01 & $40 \%$ & $56 \%$ & $42 \%$ \\
\hline $95-119$ & 5 & $141 / 78$ & 681.24 & $24 \%$ & $38 \%$ & $29 \%$ \\
\hline $120-144$ & 8 & $140 / 77$ & 442.39 & $27 \%$ & $54 \%$ & $55 \%$ \\
\hline$>145$ & 7 & $133 / 81$ & 347.04 & $28 \%$ & $14 \%$ & $57 \%$ \\
\hline p-values & 0.022 & 0.6815 & 0.289 & $<0.0001$ & $<0.0001$ & 0.00032 \\
\hline
\end{tabular}

The average RRS, average resting BP, average CAC, abnormal macrovascular (C1) percentage, abnormal microvascular (C2) percentage, and abnormal CIMT percentages for the separate groups of EFV are presented in the table. Corresponding p-values provided for statistical significance.

Table 2. EFV and biomarkers

\begin{tabular}{|c|c|c|c|}
\hline $\begin{array}{c}\text { EFV } \\
\left(\mathrm{cm}^{3}\right)\end{array}$ & $\begin{array}{c}\text { Average BNP } \\
(\mathrm{pg} / \mathrm{mL})\end{array}$ & $\begin{array}{c}\text { Average CRP } \\
(\mathrm{mg} / \mathrm{dL})\end{array}$ \\
\hline$<\mathbf{6 9}$ & 106.94 & 0.2955 \\
\hline $\mathbf{7 0 - 9 4}$ & 145.27 & 0.3016 \\
\hline $\mathbf{9 5 - 1 1 9}$ & 78.33 & 0.1947 \\
\hline $\mathbf{1 2 0 - 1 4 4}$ & 124.97 & 0.3112 \\
\hline$>\mathbf{1 4 5}$ & 57.57 & 0.6143 \\
\hline p-values & 0.3347 & 0.2759 \\
\hline
\end{tabular}

The average CRP, average BNP, and average microalbuminuria level for the separate groups of EFV are presented in the table. Corresponding p-values provided for statistical significance.

Table 3. EFV and Lipid Profile \& Blood-Sugar. The average low-density lipoprotein (LDL), average high-density lipoprotein (HDL), average triglyceride levels, average non-HDL, and average blood sugar for the separate groups EFV are presented in the table. Corresponding p-values provided for statistical significance

\begin{tabular}{|c|c|c|c|c|c|}
\hline $\begin{array}{l}\mathbf{E F V} \\
\left(\mathrm{cm}^{3}\right)\end{array}$ & $\begin{array}{l}\text { Average LDL } \\
(\mathrm{mg} / \mathrm{dL})\end{array}$ & $\begin{array}{l}\text { Average HDL } \\
(\mathrm{mg} / \mathrm{dL})\end{array}$ & $\begin{array}{l}\text { Average Triglyceride } \\
(\mathrm{mg} / \mathrm{dL})\end{array}$ & $\begin{array}{l}\text { Average non-HDL } \\
(\mathrm{mg} / \mathrm{dL})\end{array}$ & $\begin{array}{c}\text { Average Blood Sugar } \\
\qquad(\mathrm{mg} / \mathrm{dL})\end{array}$ \\
\hline$<69$ & 108.06 & 68.4 & 127 & 130.74 & 81.84 \\
\hline $70-94$ & 103.09 & 63.4 & 93 & 121.73 & 84.49 \\
\hline $95-119$ & 84.30 & 61.3 & 105 & 105.38 & 94.90 \\
\hline $120-144$ & 91.91 & 51.09 & 158 & 120.06 & 91.73 \\
\hline$>145$ & 96.93 & 45.93 & 164 & 129.71 & 108.93 \\
\hline p-values & 0.1473 & 0.0000393 & 0.0011 & 0.2265 & 0.0030 \\
\hline
\end{tabular}

important role in development of cardiovascular diseases due their pro-inflammatory properties [28]. Consequently, an increased number of macrophages, T-lymphocytes, and mast cells in epicardial fat tissue has been shown in patients with coronary artery disease by several researchers $[28,29]$. However, the effect of epicardial fat volume on the manifestation and progression of cardiovascular disease (CVD) and microvascular disease still needs to be more sufficiently and extensively explored Figure 1.

Many studies have correlated the regression of epicardial fat with weight loss and weight management, as well as routine exercise [28]. Secondly, consider using statin to reduce LDL levels below $70 \mathrm{mg} / \mathrm{dL}$ and utilizing a semaglutide for the reduction of EFV. Weekly administration of either GLP-1 receptor agonists semaglutide or dulaglutide causes a rapid, substantial, and dose-dependent reduction in EAT thickness [30]. Hopefully, these findings will be a stimulus to other investigators to better understand the role of excess epicardial fat volume in the genesis of cardiovascular disease and stimulate therapeutic interventions to prevent future cardiovascular complications.

\section{Conclusions}

Based on our data, we feel that excess EFV appears to be a significant marker for early cardiovascular structural and functional abnormalities. Accordingly, we urge all healthcare professionals that it is time to focus on early and accurate assessment of EFV which is probably the culprit for many CVD risk factors.

The majority of the female subjects in our study were of the white race, meaning that this study will need to be expanded upon with a
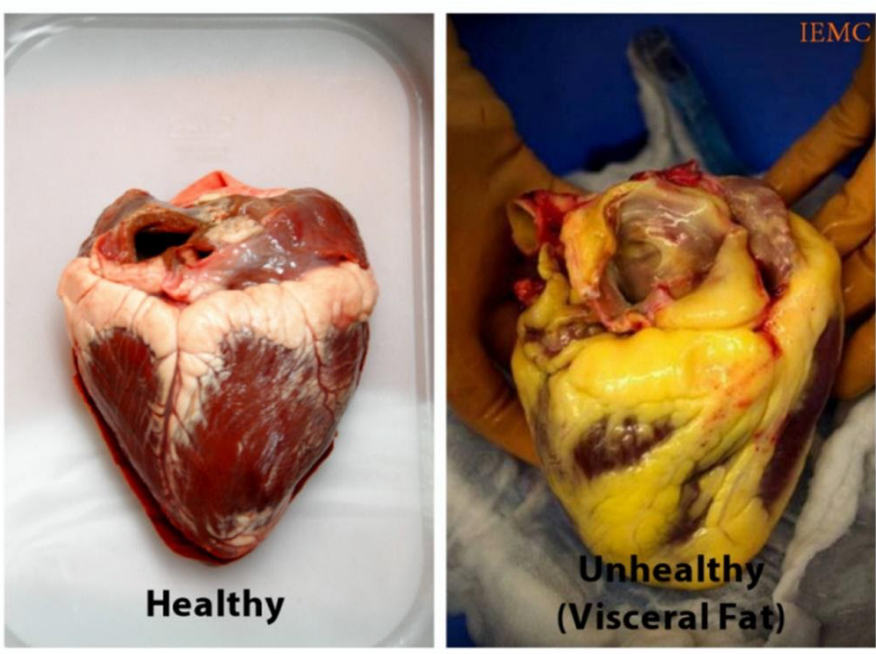

Figure 1. Healthy Heart vs. Unhealthy Heart with excess EFV

cohort of more racial diversity to further support the impact of excess EFV on cardiovascular health. Hopefully, studies in the near future will have a greater number of subjects and will be able to duplicate and confirm our findings.

\section{Declarations}

Ethics approval and consent to participate: This study was approved by the Sarasota Memorial Hospital Institutional Review Board 
(IRB) and individual patient written consent was obtained, which was a further approved by the IRB. Committee reference numbers: 08CARD-16 and 10-CARD-26.

Consent for Publication: All participants agreed to consent of publication.

Availability of Data and Material: The datasets analyzed during the current study are available from the corresponding author on reasonable request.

Funding: Cardiovascular Center of Sarasota Foundation for Education and Research, El Shahawy Family Foundation, Sarasota Memorial Healthcare Foundation,

Acknowledgements: Thanks to Sarasota Memorial Health Care Foundation, Cardiovascular Center of Sarasota Foundation for Research and Education, and El Shahawy Family Foundation for supporting this research. Thanks also to all subjects who participated in this study and to many of our colleagues for referring their patients for CV screening. Thanks also goes particularly to cardiac surgeon Dr. Thomas Kelly, who founded the Heart Surgery Program many decades ago at our institution, who made the comment to us that many of his patients who came in for Coronary Artery Bypass Graft (CABG) had excess epicardial fat. Last but not least, thanks to our staff who have been doing an excellent job and follow up, particularly Ramona Stevens, Hend Sawaf, Sharon Wetzler NP, and Lillee Izadi.

\section{References}

1. Wu Y, Zhang A, Hamilton DJ, Deng T (2017) Epicardial Fat in the Maintenance of Cardiovascular Health. Methodist DeBakey Cardiovasc J 13: 20-24. [Crossref]

2. Cabrera-Rego JO, Navarro-Despaigne D, Staroushik-Morel L, Díaz-Reyes K, LimaMartínez MM, et al. (2018) Association between endothelial dysfunction, epicardial fat and subclinical atherosclerosis during menopause. Clin Investig Arterioscler 30: 21-27. [Crossref]

3. González N, Moreno-Villegas Z, González-Bris A, Egido J, Lorenzo Ó (2017) Regulation of visceral and epicardial adipose tissue for preventing cardiovascular injuries associated to obesity and diabetes. Cardiovasc Diabetol 16: 44. [Crossref]

4. Christensen RH, Von Scholten BJ, Lehrskov LL, Rossing P, Jørgensen PG (2020) Epicardial adipose tissue: an emerging biomarker of cardiovascular complications in type 2 diabetes? Ther Adv Endocrinol Metab 11: 2042018820928824. [Crossref]

5. Kamal D, Abd El Moteleb AM, Samir R, Saeed M (2018) Epicardial fat thickness can predict severity and multivessel distribution in Egyptian patients with atherosclerotic coronary artery stenosis. Egypt Heart J 70: 323-327. [Crossref]

6. Talman AH, Psaltis PJ, Cameron JD, Meredith IT, Seneviratne SK, et al. (2014) Epicardial adipose tissue: far more than a fat depot. Cardiovasc Diagn Ther 4: 416429. [Crossref]

7. Iacobellis G, Leonetti F (2005) Epicardial Adipose Tissue and Insulin Resistance in Obese Subjects. The J of Clin Endocrinol Metabol 90: 6300-6302.

8. El Khoudary SR, Zhao Q, Venugopal V, Manson JE, Brooks MM, et al. (2019) Effects of Hormone Therapy on Heart Fat and Coronary Artery Calcification Progression: Secondary Analysis from the KEEPS Trial. J Am Heart Assoc 8: e012763. [Crossref]

9. Parsaei MS, Nabati M, Yazdani J, Bagheri B, Ghaemian A, et al. (2014) Relationship between epicardial fat and coronary microvascular dysfunction. Kardiol Pol 72: 417424. [Crossref]

10. Iacobellis G, Bianco AC (2011) Epicardial adipose tissue: emerging physiological, pathophysiological, and clinical features. Trends Endocrinol Metabol 22: 450-457. [Crossref]

11. Iacobellis G, Ribaudo MC, Assael F, Vecci E, Tiberti C, et al. (2003) Echocardiographic epicardial adipose tissue is related to anthropometric and clinical parameters of metabolic syndrome: a new indicator of cardiovascular risk. J Clin Endocrinol Metab 88: 5163-5168. [Crossref]

12. Sharma S, Ghalaut VS, Dixit R, Kumar S, George PJ (2013) Microalbuminuria and C-reactive protein as a predictor of coronary artery disease in patients of acute chest pain. J Cardiovasc Dis Res 4: 37-39. [Crossref]
13. El Khoudary SR, Venugopal V, Manson JE, Brooks MM, Santoro N, et al. (2020) Heart fat and carotid artery atherosclerosis progression in recently menopausal women: impact of menopausal hormone therapy: The KEEPS trial. Menopause 27: 255-262. [Crossref]

14. Iacobellis G, Ribaudo MC, Zappaterreno A, Iannucci CV, Leonetti F (2004) Relation between epicardial adipose tissue and left ventricular mass. Am J Cardiol 94: 10841087. [Crossref]

15. Turer AT, Scherer PE (2012) Adiponectin: mechanistic insights and clinical implications. Diabetologia 55: 2319-2326. [Crossref]

16. Wong HK, Cheung TT, Cheung BM (2012) Adrenomedullin and cardiovascular diseases. JRSM Cardiovascular Disease 1: cvd.2012.012003. [Crossref]

17. El Khoudary SR, Shields KJ, Janssen I, Hanley C, Budoff MJ, et al. (2015) Cardiovascular Fat, Menopause, and Sex Hormones in Women: The SWAN Cardiovascular Fat Ancillary Study. J Clin Endocrinol Meta 100: 3304-3312. [Crossref]

18. Cohn JN, Duprez DA, Grandits GA (2005) Arterial Elasticity as Part of a Comprehensive Assessment of Cardiovascular Risk and Drug Treatment. Hypertension 46: 217-220. [Crossref]

19. Cohn JN, Hoke L, Whitwam W, Sommers PA, Taylor AL, et al. (2003) Screening for early detection of cardiovascular disease in asymptomatic individuals. Am Heart $J$ 4: 679-685. [Crossref]

20. Duprez DA, Duval S, Hoke L, Florea N, Grandits G, et al. (2020) Early cardiovascular structural and functional abnormalities as a guide to future morbid events. Eur J Prev Cardiol 4: 2047487320901416. [Crossref]

21. Wu CF, Liu PY, Wu TJ, Hung Y, Yang SP, et al. (2015) Therapeutic modification of arterial stiffness: An update and comprehensive review. World J Cardiol 7: 742-753. [Crossref]

22. Casey Jr DE, Thomas RJ, Bhalla V, Commodore-Mensah Y, Heidenreich PA, et al (2019) 2019 AHA/ACC Clinical Performance and Quality Measures for Adults with High Blood Pressure: A Report of the American College of Cardiology/American Heart Association Task Force on Performance Measures. Circ Cardiovasc Qual Outcomes 12: e000057. [Crossref]

23. Shahawy ME, Izadi L, Sabatini A, Tucker S (2020) Quantification of Epicardial Fat Volume as a Novel Cardiovascular Risk Marker in Asymptomatic Subjects for Early Detection of Cardiovascular Disease. J Health Sci Dev 3: 5-9.

24. Forouzandeh F, Chang SM, Muhyieddeen K, Zaid RR, Trevino AR, et al. (2013) Does Quantifying Epicardial and Intrathoracic Fat with Noncontrast Computed Tomography Improve Risk Stratification Beyond Calcium Scoring Alone? Circ Cardiovasc Imaging 6: 58-66. [Crossref]

25. Dele-Michael AO, Fujikura K, Devereux RB, Islam F, Hriljac I, et al. (2013) Lef ventricular stroke volume quantification by contrast echocardiography - comparison of linear and flow-based methods to cardiac magnetic resonance. Echocardiography 30: 880-888. [Crossref]

26. Almeida S, Pelter M, Shaikh K, Cherukuri L, Birudaraju D, et al. (2020) Feasibility of measuring pericoronary fat from precontrast scans: Effect of iodinated contrast on pericoronary fat attenuation. J Cardiovasc Comput Tomogr 14: 490-494. [Crossref]

27. Gorter PM, Van Lindert AS, de Vos AM, Meijs MFL, van der Graaf Y, et al. (2008) Quantification of epicardial and peri-coronary fat using cardiac computed tomography; reproducibility and relation with obesity and metabolic syndrome in patients suspected of coronary artery disease. Atherosclerosis 197: 896-903. [Crossref]

28. Toczyłowski K, Gruca M, Baranowski M (2013) Epicardial adipose tissue and its role in cardiac physiology and disease. Postepy Hig Med Dosw (Online) 67: 584-593. [Crossref]

29. Cohn JN, El Shahawy M (2019) Monitoring Cardiovascular Disease Progression. Open J Cardiol Heart Dis 3: OJCHD.000552.2019.

30. Iacobellis G, Villasante Fricke AC (2020) Effects of Semaglutide Versus Dulaglutide on Epicardial Fat Thickness in Subjects with Type 2 Diabetes and Obesity. J Endocr Soc 4: bvz042. [Crossref]

Copyright: (C2021 El Shahawy M. This is an open-access article distributed under the terms of the Creative Commons Attribution License, which permits unrestricted use, distribution, and reproduction in any medium, provided the original author and source are credited. 